\title{
Biomarker-Driven Therapy in Metastatic Gastric and Esophageal Cancer: Real-Life Clinical Experience
}

\author{
Ofer Purim ${ }^{1,2} \cdot$ Alexander Beny ${ }^{3} \cdot$ Moshe Inbar $^{4} \cdot$ Katerina Shulman $^{5} \cdot$ Baruch Brenner $^{2,6} \cdot$ Elizabeth Dudnik $^{6}$. \\ Felix Bokstein ${ }^{4} \cdot$ Mark Temper $^{7,8} \cdot$ Dror Limon $^{4} \cdot$ Diana Matceyevsky $^{4}$. David Sarid ${ }^{4}$ - Amiel Segal ${ }^{9}$. \\ Valeriya Semenisty ${ }^{3} \cdot$ Ronen Brenner ${ }^{2,10} \cdot$ Tamar Peretz $^{7,8}$ - Efraim Idelevich ${ }^{8,11}$. Sharon Pelles-Avraham ${ }^{4}$. \\ Amichay Meirovitz ${ }^{7,8} \cdot$ Arie Figer $^{4} \cdot$ Kenneth Russell $^{12} \cdot$ Andreas Voss $^{12} \cdot$ Addie Dvir $^{13} \cdot$ Lior Soussan-Gutman $^{13}$. \\ Ayala Hubert ${ }^{7,8}$
}

Published online: 20 January 2018

(C) The Author(s) 2018. This article is an open access publication

\begin{abstract}
Background Precision treatment of cancer uses biomarker-driven therapy to individualize and optimize patient care.

Objective To evaluate real-life clinical experience with biomarker-driven therapy in metastatic gastric and esophageal cancer in Israel.

Patients and Methods This multicenter retrospective cohort study included patients with metastatic gastric or esophageal cancer who were treated in the participating institutions and underwent biomarker-driven therapy. Treatment was considered to have a benefit if the ratio between the longest progression-free survival (PFS) post biomarker-driven therapy and the last PFS before the biomarker-driven therapy was $\geq 1.3$. The null hypothesis was that $\leq 15 \%$ of patients gain such benefit.

Results The analysis included 46 patients ( $61 \%$ men; median age, 58 years; $57 \%$ with poorly-differentiated tumors). At least one actionable (i.e., predictive of response to a specific therapy) biomarker was identified for each patient. Immunohistochemistry was performed on all samples and identified 1-8 (median: 3 ) biomarkers per patient (most commonly: low TS, high TOPO1, high TOP2A). Twenty-eight patients received therapy after the biomarker analysis (1-4 lines). In the 1st line after biomarker analysis, five patients (18\%) achieved a partial response and five (18\%) stable disease; the median (range) PFS was 129 (12-1155) days. Twenty-four patients were evaluable for PFS ratio analysis; in seven (29.2\%), the ratio was $\geq 1.3$. In a one-sided exact binomial test vs. the null hypothesis, $p=0.019$; therefore, the null hypothesis was rejected.

Conclusions Our findings demonstrated that implementing biomarker-driven analysis is feasible and could provide clinical benefit for a considerable proportion $(\sim 30 \%)$ of patients with metastatic gastric or esophageal cancer.
\end{abstract}

Ofer Purim

oferhamud@gmail.com

1 Oncology Institute, Assuta Ashdod Academic Hospital, Harefua St 7, 7747629 Ashdod, Israel

2 Sackler Medical School, Tel Aviv University, Ramat Aviv, 6997801 Tel Aviv, Israel

3 Oncology Department, Rambam Health Care Campus, 6 Ha'Aliya St, 31096 Haifa, Israel

4 Division of Oncology, Tel-Aviv Sourasky Medical Center, 6 Weitzman St, 64239 Tel Aviv, Israel

5 Oncology Unit, Hillel Yaffe Medical Center, Ha-Shalom St, 38100 Hadera, Israel

6 Davidoff Cancer Center, Rabin Medical Center, Institute of Oncology, 39 Jabotinski St, 49100 Petah Tikva, Israel
7 Sharett Institute of Oncology, Hadassah Hebrew University Medical Center, 91120 Jerusalem, Israel

8 Hebrew University Hadassah Medical School, 91120 Jerusalem, Israel

9 Shaare Zedek Medical Center, The Oncology Institute, 12 Bayit St, 91031 Jerusalem, Israel

10 Oncology Department, Wolfson Medical Center, 62 HaLohamim St, 58100 Holon, Israel

11 Kaplan Medical Center, Institute of Oncology, Pasternak St, 76100 Rehovot, Israel

12 Caris Life Sciences, St. Jakobsstrasse 199, 4052 Basel, Switzerland

13 Teva Pharmaceutical Industries Ltd, 1 Hatee'na St, 60850 Shoham, Israel 


\section{Key Points}

Precision treatment of cancer identifies biomarkers (with methods such as immunohistochemistry, fluorescence/chromogenic in-situ hybridization, microarray analyses, and sequencing/next generation sequencing) that are used to tailor therapeutic regimens for each individual patient.

We investigated real-life clinical experience with biomarker-driven therapy in metastatic gastric/esophageal cancer (46 patients of whom 24 were evaluable for the clinical benefit analysis) and demonstrated that biomarker-driven therapy is feasible and could provide clinical benefit (defined as $\geq 30 \%$ longer disease-free survival after biomarker analysis vs before this analysis) for approximately $30 \%$ of patients.

\section{Introduction}

Gastric cancer is the fifth most common malignancy and the third leading cause of cancer death worldwide. Esophageal cancer is the eighth most common malignancy and the sixth leading cause of cancer death worldwide. In 2012, there were approximately 1.4 million new cases of gastric/esophageal cancers and 1.1 million deaths from these cancer types [1].

At present, there is no internationally accepted consensus regarding standard-of-care in the 1st-line metastatic setting in gastric/esophageal cancer [2]; except for 10-25\% of patients with gastric/gastroesophageal junction (GEJ) cancer whose tumors are human epidermal growth factor receptor 2 (HER2)-positive [3]. For these patients a regimen consisting of trastuzumab plus cisplatin and fluoropyrimidine has become the standard-of-care after the phase III trastuzumab for gastric/GEJ cancer (ToGA) trial demonstrated overall survival (OS) benefit for this regimen (compared with cisplatinfluoropyrimidine alone) [4], and the subsequent approval of trastuzumab in this setting. Most patients progress after 1stline treatment for metastatic gastric cancer; however, only recently did evidence supporting 2 nd-line chemotherapy treatment became available. A recent meta-analysis of randomized phase III trials by Kim et al. (2013) showed that 2nd-line treatment is associated with a significant reduction in the risk of death with both irinotecan (hazard ratio $[\mathrm{HR}]=0.55, p=$ $0.0004)$ and docetaxel (HR $=0.71, p=0.0004)$ [5]. Thus, despite recent advances in the treatment of these cancers and the demonstrated utility of targeted therapy in select patients, clinical outcomes are still poor and new treatment approaches are needed.

Precision treatment of cancer identifies tumor biomarkers to tailor therapeutic regimens for each individual patient by utilizing methodologies such as immunohistochemistry (IHC), fluorescence/chromogenic in situ hybridization (FISH/CISH), microarray analyses, and sequencing/next generation sequencing (NGS). In 2010, Von Hoff et al. demonstrated in their pilot study involving 66 patients with a variety of refractory cancers that in $27 \%$ of patients who received biomarker-driven treatment, such an approach led to progression-free survival (PFS) that was $\geq 30 \%$ longer than the last regimen on which patients progressed (before the individualized approach) [6]. Subsequently, the utility of this approach has been demonstrated in studies in specific tumor types including cancer in the breast, lung, salivary gland, esophagus, and pancreas, adenoid cystic carcinoma, thymic carcinoma, mesothelioma, and others [7-12].

The objectives of the current study were to describe reallife clinical experience with biomarker-driven therapy in patients with metastatic gastric or esophageal cancer, to characterize the molecular profile of these patients, and to evaluate their clinical outcomes.

\section{Materials and Methods}

\subsection{Study Design and Patient Population}

This multi-center retrospective study evaluated patients with metastatic gastric or esophageal cancer who were treated in the participating institutions and underwent biomarker analysis using Caris Molecular Intelligence ${ }^{\mathrm{TM}}$ (CMI) tumor profiling service (Caris Life Sciences, Irving, TX, USA) between January 2010 and March 2014. The study was approved by the institutional review boards of the participating institutions (Rabin Medical Center, Sourasky Medical Center, Rambam Health Care Campus, Hadassah Hebrew University Medical Center, and Kaplan Medical Center). All procedures performed were in accordance with the ethical standards of the institutional committee and with the 1964 Helsinki declaration and its later amendments or comparable ethical standards.

\subsection{Data Source}

Information on patients' demographic, baseline characteristics, treatments received, and clinical outcomes were collected from patients' medical records. CMI results were provided by Caris Life Sciences. Progression was determined using imaging - mostly computed tomography (CT) and positron emission tomography (PET)/CT - and biomarker analyses (CA 19-9 and carcinoembryonic antigen [CEA]). In some patients progression was determined by clinical evaluation. PFS was defined as time to progression determined as described above or death. 


\subsection{Biomarker Analysis}

CMI analyses were performed in Caris Life Sciences laboratories (Phoenix, AZ, USA) on paraffin-embedded tumor samples. The tumor samples were taken from either the primary tumor or a metastatic lesion. Biomarker analysis included six methodologies: IHC, FISH, CISH, microarray analysis, sequencing by the Sanger method, and NGS. The types of analyses performed and the specific biomarkers tested depended on the amount of tissue sample available (i.e., if the amount of the tumor sample was insufficient, the treating physician prioritized the analyses) and the specific timeframe in which the testing occurred. "Actionable" biomarkers were defined as those predictive of response to specific commercially available chemotherapeutics or biologic agents (either indicated for gastric/esophageal cancer or not), based on the available medical/scientific literature.

The molecular analyses were performed by Caris Life Sciences laboratories, as described [13, 14]. In short, IHC analysis was performed on 1 unstained slide of $4 \mu \mathrm{m}$ thickness from formalin-fixed paraffin-embedded (FFPE) tissue block with evaluable tumor present; FISH analysis was performed on two unstained slides of $4 \mu \mathrm{m}$ thickness from FFPE block with at least 100 evaluable cells present and $10 \%$ tumor; and $\mathrm{CISH}$ analysis was performed on one unstained slide of $4 \mu \mathrm{m}$ thickness from FFPE block with at least 20-100 evaluable cells present. Microarray analysis required an FFPE block with $20 \%$ tumor nuclei. NGS required an FFPE block or 15 unstained slides with at least $20 \%$ malignant origin. For IHC, thresholds were developed by Caris, except for HER2 where the American Society of Clinical Oncology (ASCO)/College of American Pathologists (CAP) scoring criteria are used. Clones used for the IHC analysis included: topoisomerase 1 (TOPO1;1D6), topoisomerase II alpha (TOP2A; 3F6), Her2/ $\mathrm{Neu}$ (4B5), excision repair cross-complementation 1 (ERCC1; 8F1), ribonucleotide reductase M1 subunit (RRM1; polyclonal), O-6-methylguanine-DNA methyltransferase (MGMT; MT23) and thymidylate synthase (TS; TS106/4H4B1). FISH analysis was used to evaluate HER-2/ neu (HER-2/CEP17 probe; HER-2/CEP17 ratio $>2.2$ was considered amplified), epidermal growth factor receptor (EGFR) (EGFR/CEP7 ratio $\geq 2$, or $\geq 15$ EGFR copies per cell in $\geq 10 \%$ of analyzed cells was considered amplified), TOP2A (TOP2A/CEP17 ratio $\geq 2.0$ was considered amplified), cMET (cMET/CEP7 ratio $\geq 5$ was considered amplified), and ALK (with an Abbott [Chicago, IL, USA] probe specific for a rearrangement of the ALK locus on chromosome 2). HER2 CISH test was performed using the INFORM DUAL HER2 ISH Assay (Ventana Medical Systems, Inc., Oro Valley, AZ, USA). For cMET and TOP2A, CISH analysis was performed using a specific probe for cMET and TOP2, respectively and a probe for the pericentromeric region of chromosome 7 (Ventana). For microarray analysis, total RNA was extracted from FFPE samples and converted to cDNA, which was subjected to a whole genome $(29 \mathrm{~K})$ microarray analysis using Illumina (San Diego, CA, USA) cDNA-mediated annealing, selection, extension, and ligation process. The expression of a subset of 88 genes was then compared to a tissue specific normal control in order to determine the relative expression ratios of these 88 target genes. Sanger sequencing was performed for selected genes ( $B R A F, K R A S, E G F R$, and $P I K 3 C A$ ), as described [14]. NGS was performed on genomic DNA isolated from FFPE tumor samples using the MiSeq platform (Illumina) as described [14].

\subsection{Statistical Analysis}

Descriptive statistics were used to summarize patient/tumor characteristics, and treatments received. Kaplan-Meier analysis was used to calculate median PFS and OS. The definition and analysis of clinical benefit followed that described by Von Hoff et al. [6]. Clinical benefit of the biomarker-driven therapy was defined as ratio of $\geq 1.3$ between the longest PFS after biomarker analysis (in any line) and the PFS on the last therapy on which the patient progressed prior to biomarker analysis (one-sided exact binomial test versus a null hypothesis of $\leq 15 \%$ of patients with PFS ratio $\geq 1.3$ was performed at a significance level of 0.05 ). The analyses were performed using JMP software, version 12.0 (SAS Institute Inc., Cary, NC, USA).

\section{Results}

\subsection{Patient Characteristics}

In total, 46 patients with gastric or esophageal cancer, who were treated in the participating institutions, underwent biomarker analysis between January 2010 and March 2014. Baseline patient and tumor characteristics are presented in Table 1. The majority of patients were males $(60.9 \%)$, and the median age was 58.4 (range, 27-78) years. Patients varied with respect of their performance status (PS) at the time of biomarker analysis; of the 30 patients for whom PS information was available, four $(13.3 \%)$ had PS 0, 15 patients (50.0\%) had PS 1, six patients $(20.0 \%)$ had PS 2, and five patients (16.7\%) had PS of 3/4. The most common tumor site was the cardia $(39.1 \%)$, and the most common histologic type was adenocarcinoma $(87.0 \%)$. The majority of patients $(56.5 \%)$ had poorly differentiated tumors. Approximately half of all patients (24 patients, $52.2 \%$ ) underwent HER2 testing on their tissue sample from their initial biopsy/surgical resection that yielded reportable results (20 patients [43.5\%] did not undergo HER2 testing, as they were diagnosed before 
Table 1 Baseline patient and tumor characteristics

\begin{tabular}{|c|c|}
\hline Characteristic & $N=46$ \\
\hline \multicolumn{2}{|l|}{ Gender, n (\%) } \\
\hline Male & $28(60.9)$ \\
\hline Female & $18(39.1)$ \\
\hline \multicolumn{2}{|l|}{ Age at diagnosis, years } \\
\hline Median (range) & $58.4(27.2-78.3)$ \\
\hline \multicolumn{2}{|l|}{ Tumor site, n (\%) } \\
\hline Cardia & $18(39.1)$ \\
\hline Gastroesophageal junction & $11(23.9)$ \\
\hline Antrum & $7(15.2)$ \\
\hline Esophagus & $7(15.2)$ \\
\hline Not available & $3(6.5)$ \\
\hline \multicolumn{2}{|l|}{ Histology, n (\%) } \\
\hline Adenocarcinoma & $40(87.0)$ \\
\hline NOS & $18(39.1)$ \\
\hline Signet ring cell & $12(26.1)$ \\
\hline Intestinal & $4(8.7)$ \\
\hline Diffuse plus signet ring cell & $3(6.5)$ \\
\hline Diffuse & $2(4.3)$ \\
\hline Intestinal plus signet ring cell & $1(2.2)$ \\
\hline Squamous carcinoma & $4(8.7)$ \\
\hline Not available & $2(4.3)$ \\
\hline \multicolumn{2}{|l|}{ Grade, n (\%) } \\
\hline Well differentiated & $1(2.2)$ \\
\hline Moderately differentiated & $11(23.9)$ \\
\hline Poorly differentiated & $26(56.5)$ \\
\hline Not available & $8(17.4)$ \\
\hline \multicolumn{2}{|l|}{ HER2 status, ${ }^{a}$ n (\%) } \\
\hline Positive & $3(6.5)$ \\
\hline Negative & $20(43.5)$ \\
\hline Equivocal & $1(2.2)$ \\
\hline Test failure & $2(4.3)$ \\
\hline Test not performed & $20(43.5)$ \\
\hline \multicolumn{2}{|l|}{ Resection of primary tumor, $n(\%)$} \\
\hline Yes & $20(43.5)$ \\
\hline No & $24(52.2)$ \\
\hline Not available & $2(4.3)$ \\
\hline \multicolumn{2}{|l|}{ Neoadjuvant therapy, n (\%) } \\
\hline Yes & $11(23.9)$ \\
\hline No & $33(71.7)$ \\
\hline Not available & $2(4.3)$ \\
\hline \multicolumn{2}{|l|}{ Adjuvant therapy, n (\%) } \\
\hline Yes & $9(19.6)$ \\
\hline No & $35(76.1)$ \\
\hline Not available & $2(4.3)$ \\
\hline \multicolumn{2}{|l|}{ Metastatic at diagnosis, n (\%) } \\
\hline Yes & $27(58.7)$ \\
\hline No & $17(37.0)$ \\
\hline Not available & $2(4.3)$ \\
\hline
\end{tabular}

${ }^{a}$ Based on the pathology report of their tumor following initial biopsy/ surgery

HER2, human epidermal growth factor receptor 2; NOS, not otherwise specified

trastuzumab was approved for this indication). Of the 24 patients for whom HER2 results were available, three $(12.5 \%)$ were found to be HER2+. Less than half of all patients $(43.5 \%)$ had resection of their primary tumor. Only a minority of patients received systemic chemotherapy in the adjuvant $(19.6 \%)$ or neoadjuvant $(23.9 \%)$ setting. More than half of the patients $(58.7 \%)$ had metastatic disease at diagnosis.

\subsection{Treatments in the Metastatic Setting Prior to Biomarker Analysis}

At the diagnosis of metastatic disease, patients had a median of two metastatic sites (range, $1-4$ ), with 25 patients (54.3\%) presenting with multiple metastatic sites. The most common metastatic sites were peritoneum (16 patients, $34.8 \%$ ), liver (13 patients, $28.3 \%$ ), and bones (seven patients, 15.2\%). Prior to undergoing biomarker analysis, patients in the metastatic setting were treated with $0-4$ (median: 1 ) lines of

Table 2 Treatment regimens received in the metastatic setting prior to biomarker analysis

\begin{tabular}{|c|c|}
\hline Treatment $^{\mathrm{a}}$ & \\
\hline First-line treatment for metastatic disease & $n=36$ \\
\hline 5-FU/capecitabine + cisplatin & $11(30.6 \%)$ \\
\hline 5FU/capecitabine + cisplatin + docetaxel & $11(30.6 \%)$ \\
\hline FOLFOX/XELOX & $4(11.1 \%)$ \\
\hline FOLFIRI & $2(5.6 \%)$ \\
\hline capecitabine + epirubicin & $2(5.6 \%)$ \\
\hline $5-\mathrm{FU}$ & $1(2.8 \%)$ \\
\hline Paclitaxel & $1(2.8 \%)$ \\
\hline Capecitabine + docetaxel + trastuzumab & $1(2.8 \%)$ \\
\hline Capecitabine + oxaliplatin + epirubicin & $1(2.8 \%)$ \\
\hline 5-FU + cisplatin + trastuzumab & $1(2.8 \%)$ \\
\hline FOLFOX + trastuzumab & $1(2.8 \%)$ \\
\hline Second-line treatment for metastatic disease & $\boldsymbol{n}=\mathbf{2 0}$ \\
\hline FOLFIRI & $7(35.0 \%)$ \\
\hline Paclitaxel & $4(20.0 \%)$ \\
\hline $5-\mathrm{FU}+$ cisplatin + docetaxel & $2(10.0 \%)$ \\
\hline Cisplatin & $1(5.0 \%)$ \\
\hline FOLFOX & $1(5.0 \%)$ \\
\hline Cisplatin + paclitaxel & $1(5.0 \%)$ \\
\hline Carboplatin + paclitaxel & $1(5.0 \%)$ \\
\hline Oxaliplatin + docetaxel & $1(5.0 \%)$ \\
\hline XELOX + trastuzumab & $1(5.0 \%)$ \\
\hline Clinical trial & $1(5.0 \%)$ \\
\hline Third-line treatment for metastatic disease & $n=10$ \\
\hline FOLFIRI & $4(40.0 \%)$ \\
\hline Paclitaxel & $4(40.0 \%)$ \\
\hline Irinotecan & $1(10.0 \%)$ \\
\hline 5-FU + epirubicin & $1(10.0 \%)$ \\
\hline Fourth-line treatment for metastatic disease & $n=3$ \\
\hline FOLFIRI & $1(33.3 \%)$ \\
\hline Cisplatin & $1(33.3 \%)$ \\
\hline Capecitabine + eribucin & $1(33.3 \%)$ \\
\hline
\end{tabular}

${ }^{\mathrm{a}}$ For two patients, information on treatment in the metastatic setting was not available

5-FU, 5- fluorouracil; FOLFIRI, 5-fluorouracil plus irinotecan; FOLFOX, 5-fluorouracil plus oxaliplatin; XELOX, capecitabine plus oxaliplatin 
therapy. Eight patients $(17.4 \%)$ had no prior therapy in the metastatic setting, $16(34.8 \%)$ had one prior line of therapy, $10(21.7 \%)$ had two prior lines, seven $(15.2 \%)$ had three prior lines, and three $(6.5 \%)$ had four prior lines of therapy for metastatic disease (for two patients, information was unavailable). Treatments received in the metastatic setting are presented in Table 2 . These treatments reflect contemporary clinical practice for treating metastatic gastric or esophageal cancer with the vast majority $(97.2 \%)$ of patients treated in the first-line metastatic setting receiving fluoropyrimidine-based therapy.

\subsection{Biomarker Analysis: Findings}

In the majority of patients (33 patients, $71.7 \%$ ), biomarker analysis was performed on samples derived from the primary tumor. In the remaining 13 patients (28.3\%), it was performed on a sample derived from a metastatic lesion (liver, four patients; peritoneum/retroperitoneum, three patients; lymph nodes, two patients, and central nervous system, chest wall, colon, and bones/joints, one patient each). Samples from each patient underwent analysis by 1-4 methodologies (median: 3 ), with approximately three quarters $(73.9 \%)$ being analyzed by two or more methodologies. Overall, the analysis identified actionable biomarkers for all 46 patients. For each patient, the analysis identified 1-17 (median: 5.5) actionable biomarkers. IHC analysis (of up to 18 biomarkers per patient) was performed on samples from all patients and identified at least one actionable biomarker for each patient (range: 1-8 biomarkers per patient; median: 3 ). FISH/CISH analysis (evaluating up to four biomarkers including ALK2p23, cMET, EGFR, HER2, and TOP2A) was performed on samples from 31 patients $(67.4 \%)$ and identified at least one actionable marker in 14 of these patient (45.2\%). Sequencing by the Sanger method (evaluating up to four genes including BRAF, EGFR, KRAS, $P I K 3 C A$ ) was performed on samples from 18 patients (39.1\%) and identified one actionable marker in 14 of these patients (77.8\%). Microarray analysis of up to 88 genes was performed on samples from 19 patients (41.3\%) and identified actionable markers in 17 patients $(89.4 \%)$. NGS of 44 genes was performed on samples from two patients and identified one actionable biomarker in one patient (PIK3CA).

For biomarkers that were IHC-tested in more than 35 patients, the most commonly identified actionable biomarker $(34 / 40,85.0 \%)$, was low/negative TS, which is associated with response to fluoropyrimidines and other folate analogs [15], followed by high TOPO1 $(27 / 40,67.5 \%)$, which is associated with response to irinotecan [16], high TOP2A (27/41, $65.9 \%$ ), which is associated with response to anthracyclines [17], and negative/low ERCC1 (21/36, 58.3\%), which is associated with response to platinum-based therapy [18] (Table 3). In addition, the expression of the receptor tyrosine kinase c-MET was IHC-assessed in 12 patients, of whom 7 (57.1\%) had negative/low c-MET levels, which is associated with lack of acquired resistance to the EGFR tyrosine kinase inhibitor (TKI) [19], and the expression of class III $\beta$-tubulin (TUBB3) was IHC-assessed in six patients of whom five $(83.3 \%)$ had negative/low TUBB3 levels, which is associated with response to taxanes [20]. In addition, HER2 testing by IHC was performed on 43 patients and HER 2 positivity was identified in two patients $(4.7 \%)$. Notably, of the three patients classified as HER2-positive in their initial workup, only one had his HER2 positivity confirmed in the current analysis, and one patient originally classified as HER2-negative was reclassified in this analysis as HER2-positive.

The most common microarray-identified actionable biomarker was overexpression of the gene coding for topoisomerase II beta $(T O P 2 B ; 10 / 19,52.6 \%)$ and overexpression of the TOP $2 A$ gene $(9 / 19,47.4 \%)$, both of which are associated with response to anthracyclines [17]. Overexpression of the gene for proto-oncogene tyrosine-protein kinase Src $(S R C)$ and overexpression of the gene for ephrin type-A receptor 2 (EPHA2), which are both associated with response to dasatinib [21], were each found in $8 / 19$ patients $(42.1 \%)$.

FISH/CISH analysis was primarily used to determine HER2 status. Samples from 28 patients were tested and the
Table 3 Common actionable biomarkers (i.e., biomarkers with positive association with response to specific chemotherapies) identified by immunohistochemistry

\begin{tabular}{llll}
\hline $\begin{array}{l}\text { Actionable } \\
\text { biomarker }\end{array}$ & $\begin{array}{l}\text { Number of patients out } \\
\text { of evaluable patients, } \mathbf{n} \mathbf{n}\end{array}$ & Frequency, $\%$ & $\begin{array}{l}\text { Drugs associated with } \\
\text { clinical benefit }\end{array}$ \\
\hline Negative/low TS & $34 / 40$ & 85.0 & $\begin{array}{l}\text { Fluoropyrimidines and other folate } \\
\text { analogs } \\
\text { Irinotecan }\end{array}$ \\
High TOPO1 & $27 / 40$ & 67.5 & Anthracyclines \\
High TOP2A & $27 / 41$ & 65.9 & Platinum-based therapy \\
Negative/low ERCC1 & $21 / 36$ & 58.3 & Gemcitabine \\
Negative/low RRM1 & $22 / 40$ & 55.0 & Temozolomide \\
Negative/low MGMT & $22 / 46$ & 47.8 & \\
\hline
\end{tabular}

Only markers that were tested in samples of at least 35 patients are included in the table

ERCC1, excision repair cross-complementation 1; MGMT, O-6-methylguanine-DNA methyltransferase; RRM1, ribonucleotide reductase M1 subunit; TOPO1, topoisomerase 1; TOP2A, topoisomerase IIA; TS, thymidylate synthase 
test yielded results in 22 patients, of whom two $(9.1 \%)$ were HER2-positive confirming their HER2 IHC results. FISH analysis was also used to test EGFR status for 22 patients (yielded results in 20 patients). Of the 20 patients, six $(30.0 \%)$ were EGFR+ (one additional patient was IHCtested for EGFR and was also found to be EGFR+). KRAS sequencing was performed on samples from 18 patients ( 16 by the Sanger method; two by NGS), of whom 17 (94.4\%) had wild-type genotype, which is associated with response to antiEGFR therapy in colorectal cancer [22].

\subsection{Treatments Post Biomarker Analysis}

Of the 46 patients in our cohort, 28 were treated with chemotherapy/biologic agents after receiving the analysis results. The remaining 18 patients included 11 patients who did not receive further treatment (beyond supportive care) due to rapidly progressing disease and a decline in PS (six of these 11 patients died within a month of receiving the biomarker analysis results; five patients died 1-3 months after receiving the results); one patient who opted not to be treated (received radiotherapy only); and six patients who were lost to followup.

Each of the 28 patients, who were treated after receiving the biomarker analysis findings, received $1-4$ lines of therapy post analysis. These treatments are listed in Table 4. In the first line after the biomarker analysis, 27 of the 28 patients $(96.4 \%)$ received biomarker-driven therapy (i.e., at least one of the agents received was consistent with the biomarker analysis findings). In the 2nd-, 3rd-, and 4th-lines of therapy after the analysis, seven of 12 patients (58.3\%), four of six (66.7\%), and three of three patients (100\%), received biomarker-driven therapy, respectively. Treatments received included those listed in the National Comprehensive Cancer Network (NCCN) guidelines for treatment of metastatic gastric and esophageal cancers (e.g., FOLFIRI, paclitaxel plus carboplatin), as well as agents not included in the list and not currently Food and Drug Administration (FDA)-approved for gastric cancer such as cetuximab, which is approved for head and neck and colorectal cancers only, or gemcitabine which is approved for ovarian, breast, pancreatic, and nonsmall cell lung cancers.

\subsection{Clinical Outcomes}

Clinical outcomes were collected for all the patients who were treated upon receiving the biomarker analysis results including patients whose treatments were not biomarker-driven: one patient in the first line; five in the second line; and two in the third line. In the first line of therapy after the biomarker analysis $(n=28)$, five patients (17.9\%) achieved a partial response (PR), five (17.9\%) stable disease (SD) (i.e., a disease control rate of 35.8\%), 16 (57.1\%) had progressive disease (PD), and
Table 4 Chemotherapy/biologic therapy regimens received after biomarker analysis

\begin{tabular}{|c|c|}
\hline First-line treatment after biomarker analysis & $n=28$ \\
\hline Doxorubicin monotherapy & $5(17.9 \%)$ \\
\hline FOLFIRI/XELIRI & $4(14.3 \%)$ \\
\hline $\mathrm{XELOX}^{\mathrm{a}}$ & $3(10.7 \%)$ \\
\hline Paclitaxel monotherapy & $2(7.1 \%)$ \\
\hline Docetaxel + trastuzumab & $1(3.6 \%)$ \\
\hline Paclitaxel + trastuzumab & $1(3.6 \%)$ \\
\hline Erlotinib monotherapy & $1(3.6 \%)$ \\
\hline Temozolomide monotherapy & $1(3.6 \%)$ \\
\hline Vinorelbine monotherapy & $1(3.6 \%)$ \\
\hline FOLFIRI + trastuzumab & $1(3.6 \%)$ \\
\hline Carboplatin + cetuximab & $1(3.6 \%)$ \\
\hline Irinotecan + cetuximab & $1(3.6 \%)$ \\
\hline $5-\mathrm{FU}+$ cisplatin + cetuximab & $1(3.6 \%)$ \\
\hline 5-FU + doxorubicin & $1(3.6 \%)$ \\
\hline 5-FU + paclitaxel & $1(3.6 \%)$ \\
\hline Capecitabine + cisplatin & $1(3.6 \%)$ \\
\hline Capecitabine + etoposide & $1(3.6 \%)$ \\
\hline Carboplatin + paclitaxel & $1(3.6 \%)$ \\
\hline Second-line treatment after biomarker analysis & $n=12$ \\
\hline Docetaxel monotherapy ${ }^{\mathrm{b}}$ & $3(25.0 \%)$ \\
\hline Paclitaxel monotherapy & $1(8.3 \%)$ \\
\hline Carboplatin monotherapy $^{\mathrm{c}}$ & $1(8.3 \%)$ \\
\hline Gemcitabine monotherapy $^{\mathrm{c}}$ & $1(8.3 \%)$ \\
\hline Irinotecan monotherapy & $1(8.3 \%)$ \\
\hline FOLFIRI & $1(8.3 \%)$ \\
\hline Gemcitabine + pemetrexed & $1(8.3 \%)$ \\
\hline Gemcitabine + trastuzumab $^{\mathrm{c}}$ & $1(8.3 \%)$ \\
\hline Capecitabine + epirubicin + cisplatin + trastuzumab & $1(8.3 \%)$ \\
\hline Capecitabine + epirubicin & $1(8.3 \%)$ \\
\hline Third-line treatment after biomarker analysis & $n=6$ \\
\hline Cisplatin monotherapy & $1(16.7 \%)$ \\
\hline Gemcitabine monotherapy ${ }^{c}$ & $1(16.7 \%)$ \\
\hline Paclitaxel monotherapy & $1(16.7 \%)$ \\
\hline Vinorelbine monotherapy $^{\mathrm{c}}$ & $1(16.7 \%)$ \\
\hline XELIRI & $1(16.7 \%)$ \\
\hline Capecitabine + lapatinib & $1(16.7 \%)$ \\
\hline Fourth-line treatment after biomarker analysis & $n=3$ \\
\hline Doxorubicin & $1(33.3 \%)$ \\
\hline Sunitinib & $1(33.3 \%)$ \\
\hline Capecitabine + etoposide & $1(33.3 \%)$ \\
\hline
\end{tabular}

${ }^{\mathrm{a}}$ In one of the XELOX-treated patients, this treatment was not biomarkerdriven

${ }^{\mathrm{b}}$ In two of the docetaxel-treated patients, this treatment was not biomarker-driven

${ }^{\mathrm{c}}$ This treatment was not biomarker-driven

5-FU, 5- fluorouracil; FOLFIRI, 5-fluorouracil /irinotecan; XELIRI, capecitabine/irinotecan; XELOX, capecitabine/oxaliplatin 
two $(7.1 \%)$ were non-evaluable (NE). The median PFS in the first line after the biomarker analysis was 129 (range, 121155 ) days. Of the 12 patients receiving 2nd-line therapy after the biomarker analysis, two (16.7\%) experienced SD, nine (75.0\%) PD, and one (8.3\%) was NE. The median PFS was 78 (range 12-294) days. Of the six patients receiving 3rd-line therapy after biomarker analysis, one (16.7\%) achieved PR, four $(66.7 \%) \mathrm{PD}$, and one (16.7\%) was NE. Fourth-line therapy after biomarker analysis was reported for only three patients (1 PD, $2 \mathrm{NE}$ ).

Twenty-four patients were included in the PFS ratio analysis (i.e., PFS could be calculated in the metastatic setting both pre- and post-biomarker analysis). In seven of these patients (29.2\%), the ratio between the longest PFS after the biomarker analysis and the PFS on their last regimen before the biomarker analysis was $\geq 1.3$ (Fig. 1). These seven patients received (after the biomarker analysis) various drugs/regimens including FOLFIRI/XELIRI (three cases), XELOX, docetaxel plus trastuzumab, and doxorubicin (one case each). Each of the agents used in these cases was biomarker-driven and all except for HER2 were identified only by IHC (Fig. 1). A onesided exact binomial test was performed versus a null hypothesis of $\leq 15 \%$ of patients with PFS ratio $\geq 1.3$. The test reached statistical significance $(p=0.019)$ and the null hypothesis was rejected. The number of treatments received for metastatic disease before biomarker analysis was similar in patients with PFS ratio of $<1.3$ and $\geq 1.3$ (mean [SD] of 1.9 [0.9] and 1.7 $[1.0]$, respectively). The median OS from diagnosis of metastatic disease (for evaluable patients who received biomarker-guided analysis; $n=27$ ) was 569 (interquartile [IQR] range, 387-761) days (Fig. 2A); the median OS from the initiation of biomarker-guided therapy was 153 (IQR range, 62-257) days (Fig. 2B).

\section{Discussion}

This study demonstrates the feasibility of implementing biomarker-driven treatment of metastatic gastric and esophageal cancers in real-life clinical practice in Israel and shows the molecular makeup of these patients. The strength of our approach lies in the combination of molecular methodologies used to identify actionable biomarkers, namely, the use of sequencing, as well as IHC, FISH, CISH, and microarray analyses. Our findings demonstrate that biomarker-driven therapy provides clinical benefit for close to a third of patients $(29.2 \%)$ in a hard-to-treat population of patients and resulted in median PFS in the first-line after biomarker analysis of 129 days, median OS (from biomarker-driven treatment) of 153 days, and median OS (from diagnosis of metastatic disease) of 569 days.

The implementation of the biomarker-driven therapy as reflected in our study is consistent with the increasing use of molecular analysis of tumors in oncology. The biomarker analysis results in the current cohort are overall consistent

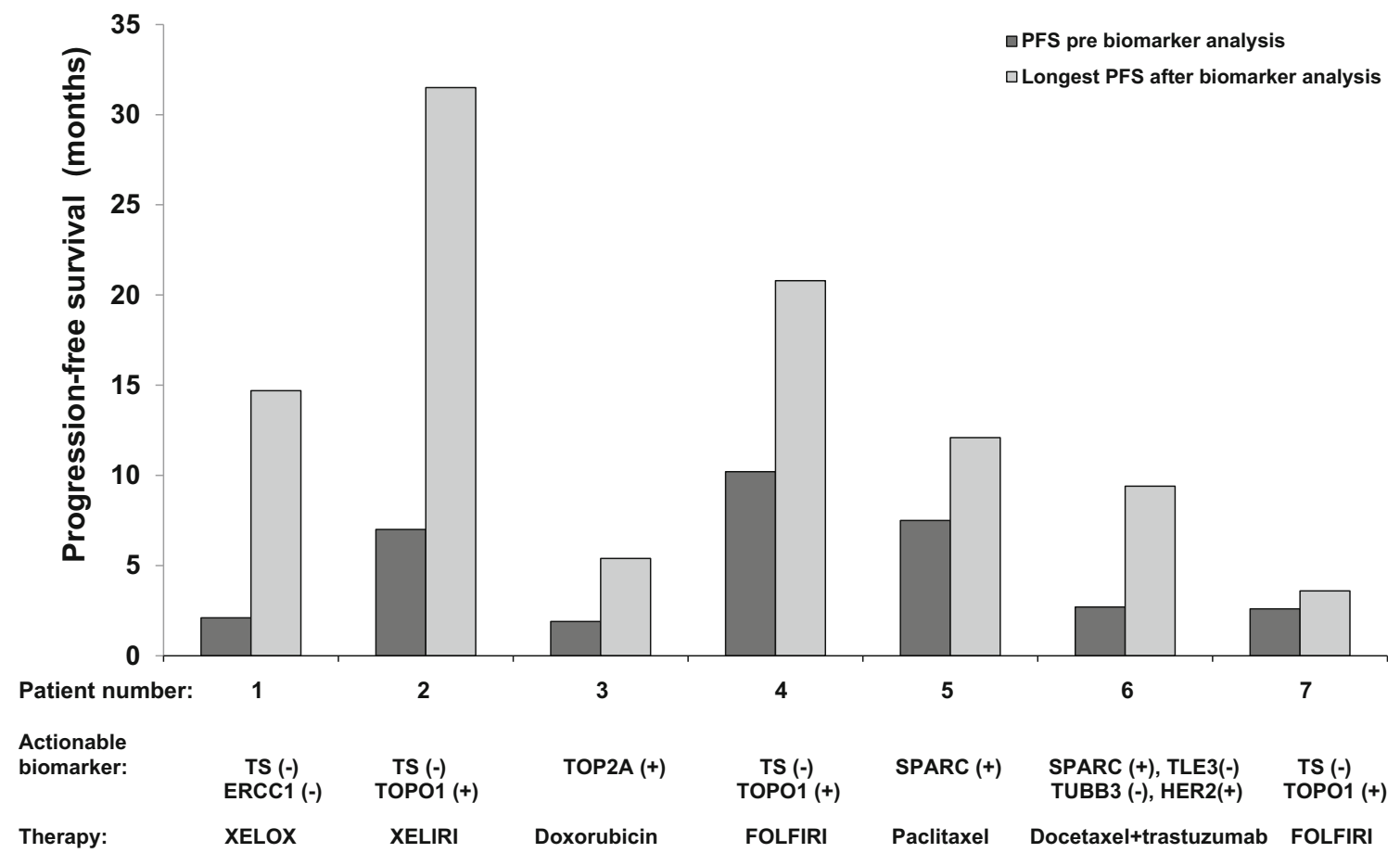

Fig. 1 Comparison between the longest PFS after knowing the biomarker analysis findings (light gray) and the PFS on the last regimen on which patients progressed before the analysis (dark gray) in seven patients for

whom the ratio between these PFS values was $\geq 1.3$. For each patient, the regimen for the longest PFS on biomarker-driven therapy and the actionable biomarker(s) supporting this treatment selection are indicated 

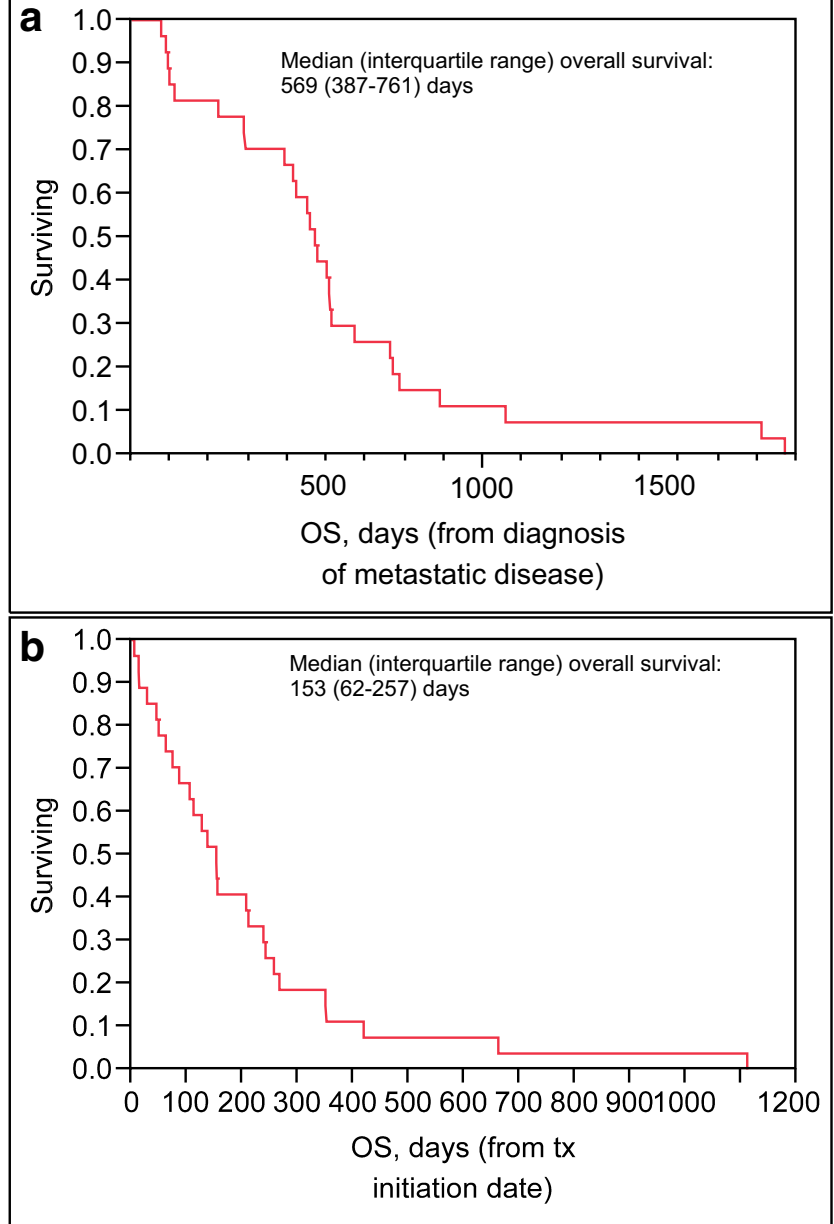

Fig. 2 Kaplan-Meier survival curve from diagnosis of metastatic disease (A) and from initiation of biomarker-driven therapy (B) for evaluable study patients who received biomarker-guided analysis $(n=27)$. Tick marks indicate censored observations

with those in a recent study involving 230 gastric cancer specimens, in which positive TOPO1 was reported for $68 \%$ of patients, negative TS for $63 \%$ of patients, high TOP2A for $60 \%$ of patients, and negative ERCC1 for $55 \%$ of patients [23]. Also, overall, our results are aligned with findings from recent studies describing the potential benefit of biomarkerdriven therapy including studies in numerous tumor types such as pancreatic, breast, salivary gland, and others [7-12]. This is, to the best of our knowledge, the first study evaluating the potential clinical value of biomarker-driven therapy in metastatic gastric and esophageal cancers.

Comparing the effectiveness of this approach to that of other approaches investigated in the metastatic gastric/ esophageal cancer setting by randomized clinical trials is challenging due to the small sample size and the heterogeneity in our cohort (e.g., biomarker-driven therapy was 1st-line treatment in $11 \%$ of our cohort, 2nd-line in $43 \%$, 3rd-line in $25 \%$, etc). Nonetheless, it is interesting to note that in the 1st-line after biomarker analysis, the observed PFS in our study was 129 days (4.3 months), which is comparable to that observed in recent phase III trials in the 2nd-line metastatic setting. For example, in the RAINBOW trial, patients randomized to receive ramucirumab plus paclitaxel had a median PFS of 4.4 months and in the TCOG GI-0801 trial patients randomized to irinotecan plus cisplatin had a median PFS of 3.8 months. Our results are potentially superior to findings from other phase III trials including the AIO study (irinotecan, 2.6 months) and REGARD (ramucirumab, 2.1 months) [24-27]. The results are also consistent with most retrospective studies conducted in the last 5 years in this setting, where the reported PFS was 3-4 months [28-32].

Our study sheds light on several issues related to the implementation of biomarker analysis in routine clinical practice. For example, in patients that received multiple lines of the therapy after the biomarker analysis, the same analysis was used to guide all therapy lines; even though the tumor may have evolved over time, still, biomarker analysis demonstrated clinical benefit. Also, our findings strongly suggest that biomarkerdriven therapy should be used in earlier rather than later lines in the advanced disease setting before PS declines and patients can no longer receive treatment. Indeed, in our study, $24 \%$ of patients were not treated after biomarker analysis due to rapidly progressing disease and a decline in PS including four of the five patients who had PS of 3 or 4 before the biomarker analysis (the patient who did receive chemotherapy relapsed and died within days of treatment initiation). Also, we observed instances where the biomarker analysis recommended one drug (e.g., irinotecan), and the physician treated with the recommended therapy, but combined it with additional drugs that were not recommended by the biomarker analysis for that particular patient (e.g., 5-FU was added to irinotecan), probably due to the known effectiveness of the drug combination.

Several barriers may impede the implementation of biomarker-driven analysis in clinical practice. The first is the cost of the test, as well as cost of the drugs recommended by the analysis (if the drugs are not indicated for the cancer type, reimbursement may be a challenge). Another barrier is related to the familiarity/confidence of physicians and patients in the approach, although this seems to be rapidly changing. In some instances, the barrier may be lack of treatment options recommended by the biomarker analysis.

Although there is a body of evidence showing the utility of the biomarker-driven therapy, the approach does have several limitations that should be considered. These include: the potential inclusion of non-tumor cells in the sample (not for IHC and FISH/CISH where tumor cells are easily differentiated, but for microarray/sequencing unless microdissection is performed); effects of the tumor microenvironment or pharmacokinetic effects on drug distribution that are not taken into account by the analysis. In addition, genomic heterogeneity should also be considered. For example, we observed three cases where the original HER2 results and the HER2 results obtained through the biomarker analysis were discordant. In 
two of these cases, the original testing was on the primary tumor and the second testing was on a metastasis. This observed discordance is consistent with a recent analysis in gastroesophageal adenocarcinoma where sequencing of primary tumors and paired metastatic lesions revealed genomic heterogeneity within primary tumors as well as between the primary tumors and the metastatic lesions [33].

Our study is limited by its retrospective design. Another key limitation is its small sample size that limits our interpretation and does not allow further analysis by clinicopathologic or molecular parameters. Additional limitations reflect its representation of real-life clinical practice. These include non-standardization of progression monitoring, heterogeneity in the testing performed, and heterogeneity in sample source (primary tumor vs. metastases).

The strength of this study stems from its true representation of real-life clinical practice experience across Israel (as it includes major medical centers treating the majority of gastric/esophageal cancer patients in Israel). The study showed that in real-life clinical practice in gastric/esophageal cancer, the biomarkerdriven therapy approach led to significant clinical benefit as defined by Von Hoff and colleagues [6], and demonstrated that biomarker analysis is a robust tool that along with other approaches could guide treatment decisions in cancer patients.

\section{Conclusions}

This study shows in real-life clinical practice that implementing biomarker-driven therapy is feasible and provides clinical benefit (PFS ratio of $\geq 1.3$ ) to close to a third of patients with metastatic gastric/esophageal cancer. Prospective studies are warranted.

Author Contributions OP and AD were involved in study design. All authors were involved in data collection. OP was involved in analysis, interpretation, and drafting the manuscript. All authors have reviewed and approved the final version of the manuscript.

\section{Compliance with Ethical Standards}

Funding This work was supported by Teva Pharmaceutical Industries Ltd. Third party medical writing and biostatistical assistance was provided by Avital Bareket-Samish, PhD (BioInsight Ltd) and was funded by Caris Life Sciences.

Conflict of Interest $\mathrm{KR}$ and $\mathrm{AV}$ are Caris Life Sciences employees. AD and LSG are Teva Pharmaceutical Industries employees. All other authors declare no conflict of interest.

Open Access This article is distributed under the terms of the Creative Commons Attribution-NonCommercial 4.0 International License (http:// creativecommons.org/licenses/by-nc/4.0/), which permits any noncommercial use, distribution, and reproduction in any medium, provided you give appropriate credit to the original author(s) and the source, provide a link to the Creative Commons license, and indicate if changes were made.

\section{References}

1. Ferlay J, Soerjomataram I, Ervik M, et al. GLOBOCAN 2012 v1.0, cancer incidence and mortality worldwide: IARC CancerBase No. 11. Available at: http://globocan.iarc.fr/Pages/fact_sheets_cancer. aspx. Accessed 30 July 2016.

2. Bilici A. Treatment options in patients with metastatic gastric cancer: current status and future perspectives. World J Gastroenterol. 2014;20:3905-15.

3. Gunturu KS, Woo Y, Beaubier N, Remotti HE, Saif MW. Gastric cancer and trastuzumab: first biologic therapy in gastric cancer. Ther Adv Med Oncol. 2013;5:143-51.

4. Bang YJ, Van Cutsem E, Feyereislova A, Chung HC, Shen L, Sawaki A, et al. Trastuzumab in combination with chemotherapy versus chemotherapy alone for treatment of HER2-positive advanced gastric or gastro-oesophageal junction cancer (ToGA): a phase 3, open-label, randomised controlled trial. Lancet. 2010;376:687-97.

5. Kim HS, Kim HJ, Kim SY, Kim TY, Lee KW, Baek SK, et al. Second-line chemotherapy versus supportive cancer treatment in advanced gastric cancer: a meta-analysis. Ann Oncol. 2013;24: 2850-4.

6. Von Hoff DD, Stephenson JJ Jr, Rosen P, Loesch DM, Borad MJ, Anthony S, et al. Pilot study using molecular profiling of patients tumors to find potential targets and select treatments for their refractory cancers. J Clin Oncol. 2010;28:4877-83.

7. Jameson GS, Petricoin E, Sachdev JC, Liotta LA, Loesch D, Anthony SP, et al. A pilot study utilizing molecular profiling to find potential targets and select individualized treatments for patients with metastatic breast cancer. J Clin Oncol. 2013;31(suppl; abstr TPS11123).

8. Ramanathan RK, Barrett M, Weiss GJ, Posner R, Rajeshkumar NV, Jameson G, et al. Phase II study of therapy selected by molecular profiling in patients with previously treated metastatic pancreatic cancer. A study of the stand up to cancer (SU2C) consortium. Cancer Res. 2012;72(8 suppl 1):Abstract LB-221.

9. Popovtzer A, Sarfaty M, Limon D, Marshack G, Perlow E, Dvir A, et al. Metastatic salivary gland tumors: a single-center study demonstrating the feasibility and potential clinical benefit of molecularprofiling-guided therapy. Biomed Res Int. 2015;2015:614845.

10. Epelbaum R, Shacham-Shmueli E, Klein B, Agbarya A, Brenner B, Brenner R, et al. Molecular profiling-selected therapy for treatment of advanced pancreaticobiliary cancer: a retrospective multicenter study. Biomed Res Int. 2015;2015:681653.

11. Kris MG, Johnson BE, Berry LD, Kwiatkowski DJ, Iafrate AJ, Wistuba II, et al. Using multiplexed assays of oncogenic drivers in lung cancers to select targeted drugs. JAMA. 2014;311:19982006.

12. Park HS, Lim SM, Kim S, Kim S, Kim HR, Kwack K, et al. Pilot study of a next-generation sequencing-based targeted anticancer therapy in refractory solid tumors at a Korean institution. PLoS One. 2016;11:e0154133.

13. Abbosh P, Sundararajan S, Millis SZ, Hauben A, Reddy S, Geynisman DM, et al. Molecular and genomic profiling to identify actionable targets in chromophobe renal cell cancer. Eur Urol Focus. 2017.

14. Borazanci E, Millis SZ, Kimbrough J, Doll N, Von Hoff D, Ramanathan RK. Potential actionable targets in appendiceal cancer detected by immunohistochemistry, fluorescent in situ hybridization, and mutational analysis. J Gastrointest Oncol. 2017;8:164-72.

15. Johnston PG, Lenz HJ, Leichman CG, Danenberg KD, Allegra CJ, Danenberg PV, et al. Thymidylate synthase gene and protein expression correlate and are associated with response to 5-fluorouracil in human colorectal and gastric tumors. Cancer Res. 1995;55: $1407-12$. 
16. Kostopoulos I, Karavasilis V, Karina M, Bobos M, Xiros N, Pentheroudakis G, et al. Topoisomerase I but not thymidylate synthase is associated with improved outcome in patients with resected colorectal cancer treated with irinotecan containing adjuvant chemotherapy. BMC Cancer. 2009;9:339.

17. O'Malley FP, Chia S, Tu D, Shepherd LE, Levine MN, Huntsman $\mathrm{D}$, et al. Topoisomerase II alpha protein and responsiveness of breast cancer to adjuvant chemotherapy with CEF compared to CMF in the NCIC CTG randomized MA.5 adjuvant trial. Breast Cancer Res Treat. 2011;128:401-9.

18. Huang MY, Tsai HL, Lin CH, Huang CW, Ma CJ, Huang CM, et al. Predictive value of ERCC1, ERCC2, and XRCC1 overexpression for stage III colorectal cancer patients receiving FOLFOX-4 adjuvant chemotherapy. J Surg Oncol. 2013;108:457-64.

19. Zucali PA, Ruiz MG, Giovannetti E, Destro A, Varella-Garcia M, Floor K, et al. Role of cMET expression in non-small-cell lung cancer patients treated with EGFR tyrosine kinase inhibitors. Ann Oncol. 2008;19:1605-12.

20. Hwang JE, Hong JY, Kim K, Kim SH, Choi WY, Kim MJ, et al. Class III beta-tubulin is a predictive marker for taxane-based chemotherapy in recurrent and metastatic gastric cancer. BMC Cancer. 2013;13:431.

21. Wang XD, Reeves K, Luo FR, Xu LA, Lee F, Clark E, et al. Identification of candidate predictive and surrogate molecular markers for dasatinib in prostate cancer: rationale for patient selection and efficacy monitoring. Genome Biol. 2007;8:R255.

22. Misale S, Yaeger R, Hobor S, Scala E, Janakiraman M, Liska D, et al. Emergence of KRAS mutations and acquired resistance to anti-EGFR therapy in colorectal cancer. Nature. 2012;486:532-6.

23. Miura JT, Johnston FM, Thomas J, George B, Eastwood D, Tsai S, et al. Molecular profiling in gastric cancer: examining potential targets for chemotherapy. J Surg Oncol. 2014;110:302-6.

24. Wilke H, Muro K, Van Cutsem E, SC O, Bodoky G, Shimada Y, et al. Ramucirumab plus paclitaxel versus placebo plus paclitaxel in patients with previously treated advanced gastric or gastrooesophageal junction adenocarcinoma (RAINBOW): a doubleblind, randomised phase 3 trial. Lancet Oncol. 2014;15:1224-35.
25. Higuchi K, Tanabe S, Shimada K, Hosaka H, Sasaki E, Nakayama $\mathrm{N}$, et al. Biweekly irinotecan plus cisplatin versus irinotecan alone as second-line treatment for advanced gastric cancer: a randomised phase III trial (TCOG GI-0801/BIRIP trial). Eur J Cancer. 2014;50: 1437-45.

26. Thuss-Patience PC, Kretzschmar A, Bichev D, Deist T, Hinke A, Breithaupt K, et al. Survival advantage for irinotecan versus best supportive care as second-line chemotherapy in gastric cancer-a randomised phase III study of the Arbeitsgemeinschaft Internistische Onkologie (AIO). Eur J Cancer. 2011;47:2306-14.

27. Fuchs CS, Tomasek J, Yong CJ, Dumitru F, Passalacqua R, Goswami C, et al. Ramucirumab monotherapy for previously treated advanced gastric or gastro-oesophageal junction adenocarcinoma (REGARD): an international, randomised, multicentre, placebo-controlled, phase 3 trial. Lancet. 2014;383:31-9.

28. Bohanes P, Courvoisier DS, Perneger TV, Morel P, Huber O, Roth AD. Survival predictors for second-line chemotherapy in Caucasian patients with metastatic gastric cancer. Swiss Med Wkly. 2011;141: w13249.

29. Maugeri-Sacca M, Pizzuti L, Sergi D, Barba M, Belli F, Fattoruso $\mathrm{S}$, et al. FOLFIRI as a second-line therapy in patients with docetaxel-pretreated gastric cancer: a historical cohort. J Exp Clin Cancer Res. 2013;32:67.

30. Kucukzeybek Y, Dirican A, Erten C, Somali I, Can A, Demir L, et al. Second-line irinotecan after cisplatin, fluoropyrimidin and docetaxel for chemotherapy of metastatic gastric cancer. Asian Pac J Cancer Prev. 2012;13:2771-4.

31. Kanagavel D, Pokataev IA, Fedyanin MY, Tryakin AA, Bazin IS, Narimanov MN, et al. A prognostic model in patients treated for metastatic gastric cancer with second-line chemotherapy. Ann Oncol. 2010;21:1779-85.

32. Pistelli M, Scartozzi M, Bittoni A, Galizia E, Berardi R, Cascinu S. Second-line chemotherapy with irinotecan, 5-fluorouracil and leucovorin (FOLFIRI) in relapsed or metastatic gastric cancer: lessons from clinical practice. Tumori. 2011;97:275-9.

33. Pectasides E, Stachler MD, Derks S, Liu Y, Maron S, Islam M, et al. Genomic heterogeneity as a barrier to precision medicine in gastroesophageal adenocarcinoma. Cancer Discov. 2017. 